\title{
Sperm miRNAs - potential mediators of bull age and early embryo development
}

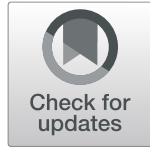

Chongyang Wu', Patrick Blondin², Christian Vigneault², Rémi Labrecque ${ }^{2}$ and Marc-André Sirard ${ }^{1 *}$ (D)

\begin{abstract}
Background: Sperm miRNAs were reported to regulate spermatogenesis and early embryonic development in some mammals including bovine. The dairy cattle breeding industry now tends to collect semen from younger bulls under high selection pressure at a time when semen quality may be suboptimal compared to adult bulls. Whether the patterns of spermatic miRNAs are affected by paternal age and/or impact early embryogenesis is not clear. Hence, we generated small non-coding RNA libraries of sperm collected from same bulls at 10, 12, and 16 months of age, using 16 months as control for differential expression and functional analysis.

Results: We firstly excluded all miRNAs present in measurable quantity in oocytes according to the literature. Of the remaining miRNAs, ten sperm-borne miRNAs were significantly differentially expressed in younger bulls (four in the 10 vs 16 months contrast and six in the 12 vs 16 months contrast). Targets of miRNAs were identified and compared to the transcriptomic database of two-cell embryos, to genes related to two-cell competence, and to the transcriptomic database of blastocysts. Ingenuity pathway analysis of the targets of these miRNAs suggested potential influence on the developmental competence of two-cell embryos and on metabolism and protein synthesis in blastocysts.
\end{abstract}

Conclusions: The results showed that miRNA patterns in sperm are affected by the age of the bull and may mediate the effects of paternal age on early embryonic development.

Keywords: Paternal age, Sperm miRNAs, Embryonic development, Dairy bull, Epigenetics

\section{Background}

With genomic selection technologies, dairy cattle breeding companies can now use bulls for reproductive purpose at early pubertal stages with high reliability [1]. Compared to waiting until five years of age for progeny testing, this practice dramatically increases the speed of genetic gain by reducing generation intervals.

Puberty in bulls, which is initiated by systemic information of the neuroendocrine system, is defined as the age when the first ejaculation containing over $50 \times 10^{6}$ million

\footnotetext{
* Correspondence: Marc-Andre.Sirard@fsaa.ulaval.ca

'Centre de Recherche en Reproduction, Développement et Santé Intergénérationnelle (CRDSI), Département des Sciences Animales, Faculté des Sciences de l'Agriculture et de l'Alimentation, Université Laval, Québec, Québec, Canada

Full list of author information is available at the end of the article
}

spermatozoa with at least $10 \%$ progressive motility is observed [2]. It is thus predictable that semen collected from younger bulls has lower sperm count, lower motility, and results in lower IVF rates compared to sperm collected from mature bulls [3]. A lack of functional mitochondria in sperm of prepubertal bulls was also observed which may explain the reduced motility of sperm at this age [3]. It would be interesting to identify factors that could potentially be affected by paternal age in order to better understand the effects of using younger bulls on embryo and offspring development.

During fertilization, sperm transfer not only the paternal genome, but also a variety of epigenetic information, including DNA methylation, histone retention and modifications, as well as small non-coding RNAs (sncRNAs) [4]. In contrast to genetic information which is relatively 
stable, epigenetic factors conveyed by male gametes are more vulnerable to environmental and physiological changes and are able to affect offspring health transgenerationally $[5,6]$. Increasing evidence suggests that DNA methylation patterns, miRNAs, and modifications of retained histones are related to bull fertility status [7-10]. Moreover, differentially methylated regions (DMRs) were observed in sperm of bulls at different pubertal stages $[3,11$, 12]. However, following DNA methylation reprogramming during embryogenesis, only a few DMRs were retained [11], suggesting that other epigenetic factors, such as sncRNAs, may potentially function as paternal transgenerational inheritance factors.

Sperm sncRNAs were initially regarded as residues of spermatogenesis. However, recent studies discovered that sncRNAs, such as miRNAs and tsRNAs, are sensitive to environmental, metabolic, and psychological stresses $[6,13,14]$. Such programming could then transgenerationally impact preimplantation embryos [15] and even offspring development [16]. Sperm cells carrying different miRNA and/or endo-siRNA deficiencies in Dicer and Drosha conditional knockout mice could still fertilize oocytes but the developmental potential of the resulting embryos was significantly reduced [17]. Individual miRNA studies also concluded that sperm-borne miR-34c is required for the first cell division in mouse embryos via targeting of the Bcl-2 mRNA [18]. In bovine spermatozoa, the microRNA pattern was demonstrated by deep sequencing [19]. However, whether the expression pattern of sperm-borne sncRNAs varies during the sexual maturation of young bulls is not clear and their functions in early embryonic development have not been described yet.

The objective of this study was to assess the potential differences between small non-coding RNAs in semen of bulls collected at 10,12, and 16 months of age (pre-, peri-, and post-pubertal stages, respectively). More than a thousand bovine miRNAs were identified, and ten sperm-borne miRNAs were significantly differentially expressed in younger bulls. These miRNA targets are associated with developmental and metabolic pathways such as TGF- $\beta$, PI3K/AKT, oxidative phosphorylation and mitochondrial dysfunction in pre-implantation embryos, thus may mediate paternal age effects on early embryonic development.

\section{Results}

\section{Bovine sperm small RNA purification}

Semen was collected from the same four bulls at the ages of 10,12 , and 16 months, representing the pre-, peri- and post-pubertal stages, respectively. Semen quality was analyzed, bulls of 10 months old ejaculated significantly less sperm ( $p$-value $<0.01)$ and had a tendency of lower concentration $(p$-value $=0.19)$ and lower post-thaw progressive motility rate compared to 12 and 16 months old ( $p$-value $=$ 0.21 ), while no difference was observed in total motility among three ages (Supplementary Material, Fig. S1).

An average of $87 \mathrm{ng}$ total RNA with RIN of $2.45 \pm$ 0.015 was extracted from five straws for each age group (Supplementary Material, Table S1). Bovine spermatozoa from semen collected at different pubertal stages displayed similar overall RNA profiles. The RNA profile of bull \#06622 at 10 months old is shown as an example: most fragments were shorter than 200 nt illustrating the sperm specific short-length RNA pattern (Fig. 1a and b). During library construction, sperm total RNA was ligated to adapters $125 \mathrm{nt}$ long and reverse transcribed. Following amplification, the cDNA libraries were purified by TBE gel, and fragments between 145 and $400 \mathrm{nt}$, which represented small non-coding RNAs, were cut for sequencing (Fig. 1c).

\section{Small non-coding RNA patterns in spermatozoa from bulls at different pubertal stages}

A total of 4625 and 4615 unique sequences were identified in the 10 vs 16 months and the 12 vs 16 months contrasts, respectively, including $1080 \mathrm{miR}$ NAs and 60 tsRNAs for the former as well as 1075 miRNAs and 60 tsRNAs for the latter group, which were mapped to the bovine database (Fig. 2a and b). Differential expression analysis for the four bulls compared to themselves at a different age resulted in two miRNAs significantly overexpressed and four miRNAs underexpressed in spermatozoa of bulls at 10 months compared to 16 months of age. Similarly, three miRNAs and one tsRNA were upregulated while five miRNAs were down regulated at 12 months compared to 16 months of age. After filtering out the small non-coding RNAs known to be present in maternal gametes $[20,21]$, ten differentially expressed miRNAs were selected from the two contrasts for further functional analysis (Table 1, and Supplementary Material, Table S2).

\section{Paternal age effects on two-cell embryos}

The predicted target genes of these miRNAs were obtained from TargetScan (release 7.2) [22] to explore the potential paternal age effects on early embryo development. Ingenuity pathway analysis (IPA) of target genes expressed in two-cell embryos according to our previous study [23] indicated that the top five canonical pathways affected by paternal age were mainly related to metabolic and developmental pathways (Table 2). For example, TGF- $\beta$ signaling and Rho family GTPase signaling were activated in the $10 \mathrm{vs}$ 16 months group, while PI3K/AKT signaling, Insulin receptor signaling, and AMPK signaling were activated and PTEN signaling was inhibited in the 12 vs 


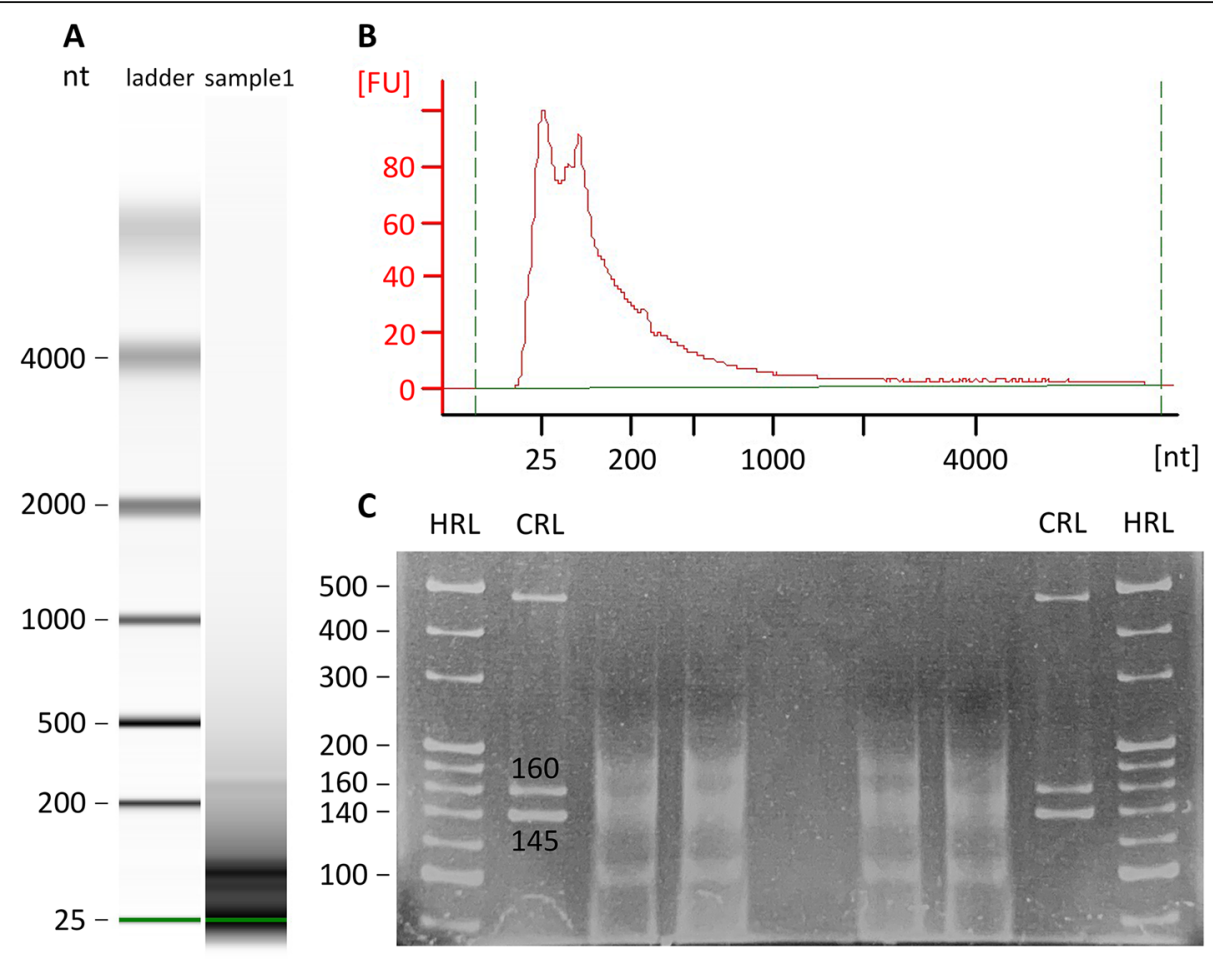

Fig. 1 RNA patterns of sperm from bull \#06622 at 10 months old. a, b: Bioanalyzer results of total RNA extracted from sperm. c: Small RNA libraries with adapters of $125 \mathrm{nt}$ (small noncoding RNAs as well as miRNAs were cut and purified for sequencing). HRL: High Resolution Ladder. CRL: Custom RNA Ladder. Summary of all RNA patterns of sperm was shown in Supplementary Material (Fig. S2)

16 months group. Insulin-like growth factor -1 signaling activation was included in the top five list for each contrast. Thus, miRNAs from sperm of younger bulls may potentially impact the metabolism and development of two-cell embryos.
Paternal age effects on developmental competence of two-cell embryos

Early embryonic cleavage kinetics is one of the most important indicators of developmental competence with embryos cleaving sooner following fertilization possessing

\section{A 10 vs 16 months}

60

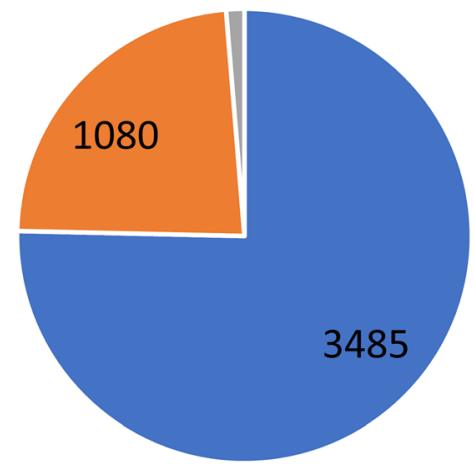

others

miRNA $\square$ tsRNA

\section{B 12 vs 16 months}

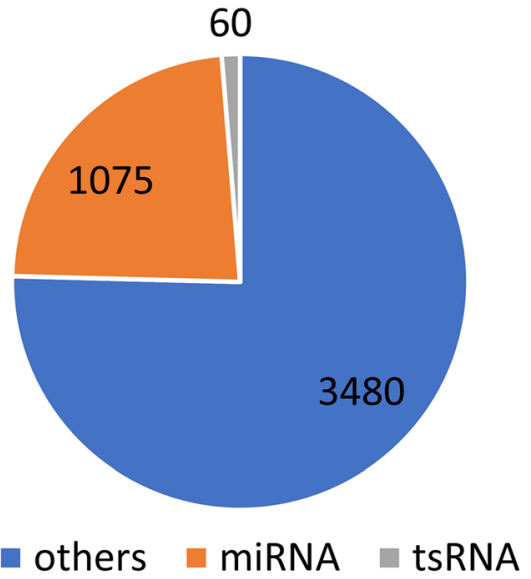

Fig. 2 Pie charts of RNA distribution in sperm. a: The distribution of RNAs that are expressed in sperm from bulls at 10 vs 16 months. b: The distribution of RNAs that are expressed in sperm from bulls at 12 vs 16 months 
Table 1 Differentially expressed sperm-borne miRNAs in two contrasts

\begin{tabular}{|c|c|c|c|c|c|}
\hline \multicolumn{3}{|l|}{10 vs 16 months } & \multicolumn{3}{|l|}{12 vs 16 months } \\
\hline Candidate miRNA & Fold Change & $p$-value & Candidate miRNA & Fold Change & $p$-value \\
\hline bta-mir-19b & 2.20 & 0.0029 & bta-mir-759 & 4.99 & 0.0008 \\
\hline bta-mir-133a & 1.89 & 0.0354 & bta-mir-2284z & 4.44 & 0.0213 \\
\hline bta-mir-2285b & -3.45 & 0.0273 & bta-mir-34b & 2.14 & 0.0227 \\
\hline \multirow[t]{3}{*}{ bta-mir-2405 } & -1.52 & 0.0436 & bta-miR-2334 & -4.35 & 0.0230 \\
\hline & & & bta-mir-2285e & -2.44 & 0.0105 \\
\hline & & & bta-mir-302a & -2.38 & 0.0401 \\
\hline
\end{tabular}

higher developmental capacity [24, 25]. A data set was previously obtained by comparing the mRNA content of two-cell embryos that had divided shortly after fertilization (competent) to later dividing embryos (less competent). Transcripts present at the two-cell stage are either translated rapidly between the two- and eight-cell stages, or simply degraded by the eight-cell stage, therefore creating a two to three-day window for being targeted by sperm-borne or other miRNAs [26-28]. In our previous study, we identified 1368 gene transcripts that were differentially expressed in fast- vs slow-cleaving two-cell embryos [23]. In the present study, 424 and 332 of these 2-cell developmental competence related genes were targets of differentially expressed miRNAs in sperm from bulls at 10 vs 16 months, and 12 vs 16 months of age, respectively. Furthermore, IPA revealed that the top five canonical pathways of candidate genes targeted by differentially expressed miRNAs in the 10 vs 16 months group were related to embryonic stem cell pluripotency, biosynthetic process, and thrombin signaling (Table 3). Meanwhile, in the 12 vs 16 months group, the top five canonical pathways activated by the differentially expressed miRNAs were PI3K signaling, iCOS-iCOSL signaling, inhibition of angiogenesis by TSP1, non-small cell lung cancer signaling, and telomerase signaling (Table 3 ). In this case, spermatozoa ejaculated from younger bulls could influence the metabolism, pluripotency, and immune response related pathways to impact the developmental capacity of the two-cell embryos immediately or between the two- and eight-cell stages.

\section{Paternal age effects on blastocysts}

Due to the relative short half-life of miRNAs, a direct effect of sperm-borne miRNAs at the blastocyst stage is questionable. However, it is possible that the miRNA targets will transmit the paternal age effects to later stages or create a context that will have an impact a few cell cycles later at the blastocyst stage. To explore these possibilities, we used the transcriptomic data of blastocysts produced in vitro from sperm of younger bulls [29]. In that study, we used IPA to predict the upstream regulators of the differentially expressed genes (DEGs) in blastocysts from younger bulls in a very similar age design as the present study. Among the upstream regulators previously identified, three were targets of miRNAs that were differentially expressed at 10 vs 16 months, and four were targets of miRNAs differentially expressed at 12 vs 16 months (Table 4). Interestingly, two of the upstream regulators (RICTOR and KDM5A) were common to the two groups. IPA of the downstream targets of these common regulators demonstrated that four of the top five canonical pathways were common to the two contrasts, e.g. oxidative phosphorylation, mitochondrial dysfunction, EIF2 signaling, and sirtuin signaling pathway (Table 5). These pathways were all closely related to the metabolism of blastocysts.

\section{Discussion}

Function of sperm miRNA in early embryo development The function of sperm-borne miRNAs was ignored for a long time as they were thought to simply be remnants or byproducts of spermatogenesis. Recently, promising

Table 2 Top five canonical pathways assembled from targets of differentially expressed sperm-borne miRNAs in two-cell embryos

\begin{tabular}{|c|c|c|c|c|c|}
\hline \multicolumn{3}{|l|}{10 VS 16 months } & \multicolumn{3}{|l|}{12 VS 16 months } \\
\hline Canonical Pathways & $-\log (p$-value $)$ & z-score & Canonical Pathways & $-\log (p$-value $)$ & z-score \\
\hline TGF- $\beta$ Signaling & 11.4 & 4.95 & PI3K/AKT Signaling & 10.1 & 2.402 \\
\hline Signaling by Rho Family GTPases & 10.8 & 6.565 & PTEN Signaling & 7.39 & -3.43 \\
\hline Huntington's Disease Signaling & 10.1 & 2.333 & Insulin Receptor Signaling & 6.67 & 2 \\
\hline IGF-1 Signaling & 9.59 & 4.226 & IGF-1 Signaling & 6.31 & 3.266 \\
\hline Cardiac Hypertrophy Signaling & 9.13 & 5.515 & AMPK Signaling & 6.21 & 2.596 \\
\hline
\end{tabular}

Note: $\mathrm{A}$ Z-score $\geq 2$ means predicted activation, while a $\mathrm{z}$-score $\leq-2$ predicts inhibition 
Table 3 Top five canonical pathways assembled from targets of differentially expressed sperm-borne miRNAs correlated with twocell embryo competence

\begin{tabular}{|c|c|c|c|c|c|}
\hline \multicolumn{3}{|l|}{10 VS 16 months } & \multicolumn{3}{|l|}{12 VS 16 months } \\
\hline Canonical Pathways & $-\log (p$-value $)$ & z-score & Canonical Pathways & $-\log (p$-value $)$ & z-score \\
\hline Mouse Embryonic Stem Cell Pluripotency & 4.81 & 2.111 & PI3K Signaling in B Lymphocytes & 3.62 & 3 \\
\hline Superpathway of Inositol Phosphate Compounds & 3.63 & 3.207 & iCOS-iCOSL Signaling in T Helper Cells & 3.22 & 2.646 \\
\hline $\begin{array}{l}\text { Role of NANOG in Mammalian Embryonic Stem } \\
\text { Cell Pluripotency }\end{array}$ & 3.61 & 2.646 & Inhibition of Angiogenesis by TSP1 & 2.79 & 2 \\
\hline Thrombin Signaling & 3.41 & 2.714 & Non-Small Cell Lung Cancer Signaling & 2.68 & 2.236 \\
\hline 3-phosphoinositide Biosynthesis & 3.21 & 2.887 & Telomerase Signaling & 2.61 & 2.449 \\
\hline
\end{tabular}

Note: A Z-score $\geq 2$ means predicted activation, while a z-score $\leq-2$ predicts inhibition

results were obtained regarding the regulation of spermborne miRNAs in early embryonic development $[17,18$, 30]. One of the six miRNAs (miR-34c) expressed in sperm and zygotes, but not in oocytes and preimplantation embryos, was required for the first cell division in vitro [18]. However, in vivo research using miR-34b/c and miR-449 double knockout (miR-dKO) mice demonstrated that the two miRNA clusters were indispensable for spermatogenesis and fertility, but not for fertilization or preimplantation development [30]. The conflicting results were mainly due to the off-target effects of the inhibitor of miR-34c used in the former study, indicating that other sperm-borne miRNAs might be involved in early embryonic development. This assumption was further verified by germline-specific Dicer and Drosha conditional knockout in mice, which resulted in failure of zygotic genome activation and embryonic development [17]. One member of this miRNA family, miR-34b, was differentially expressed in the sperm of young bulls; however, the blastocyst rates were not significantly affected [29], indicating that this miRNA does not have a lethal influence on bovine embryos. Molecular and cellular function analysis using IPA predicts that the differentially expressed sperm-borne miRNAs from young bulls will potentially influence the gene expression, cellular assembly and organization, and cellular function and maintenance at 2-cell stage, while later at blastocysts stage, these miRNAs will be likely to affect RNA damage and repair, protein synthesis, and small molecule biochemistry (Fig. 3). As in other species, it is therefore quite possible that the dominant biofunctions of these miRNAs is to carry paternal epigenetic effects.

\section{sncRNAs as epigenetic inheritance factors}

Epigenetic factors, including DNA methylation, noncoding RNAs, histone modification, and others, are more sensitive to environmental conditions compared to genetic information. Epigenetic information in sperm was proven to translate the environmental experiences of the father who could then transfer these acquired traits to his progeny [31]. In a previous study from our lab, sperm from prepubertal bulls were characterized to bear a different DNA methylation pattern compared to sperm from post-pubertal bulls [11]. Moreover, in the mouse, the global reprogramming during early embryonic development does not erase all methylation marks from the sperm, leaving a few regions for inter or transgenerational inheritance [32]. In our study mentioned above, there were over 2600 differentially methylated regions between sperm of bulls at 10 and 16 months of age, including one of them located on an imprinted gene [11]. Recently, contradictory evidence cast some doubts on whether sperm DNA methylation is either the primary or the persistent epigenetic carrier of paternally acquired traits [33, 34]. Hence, other epigenetic factors such as sperm-borne sncRNAs are gaining more and more attention as transgenerational inheritance factors from the paternal side. The direct causal relationship between sperm RNAs and paternally inherited characteristics was first reported in a study in which total sperm RNA from traumatized mice was injected into wild type zygotes resulting in offspring with altered behavior and metabolism [14]. Catenin $\beta 1$ (Ctnnb1), which is involved in stress pathways, was validated as a target of miR-375 which was differentially expressed in mentally stressed

Table 4 Upstream regulators of the blastocyst transcriptome that are targets of sperm borne miRNAs

\begin{tabular}{llll}
\hline $\begin{array}{l}\text { Upstream regulators } \\
\text { in } \mathbf{1 0} \text { vs } \mathbf{1 6} \text { months }\end{array}$ & Downstream targets & $\begin{array}{l}\text { Upstream regulators } \\
\text { in } \mathbf{1 2} \text { vs } \mathbf{1 6} \text { months }\end{array}$ & Downstream targets \\
\hline RICTOR & ATP5MF, ATP6V0A4, ATP6V1D, etc. 24 genes & RICTOR & ATP5PD, ATP6V0D2, COX7A2L, etc. 18 genes \\
KDM5A & ACTC1, ATP6V1D, ATP6V1F, etc. 15 genes & STK11 & ATIC, AVIL, CCNA2, etc. 14 genes \\
CREB1 & CHRNA1, CNN1, CSRP2, etc. 21 genes & KDM5A & COQ7, MCM3, MYL4, etc. 7 genes \\
& & AREG & CCNA2, CENPF, PRC1, etc. 5 genes \\
\hline
\end{tabular}


Table 5 Top five canonical pathways potentially affected by bull age-related sperm-borne miRNAs in blastocysts

\begin{tabular}{|c|c|c|c|}
\hline \multicolumn{2}{|l|}{10 VS 16 months } & \multicolumn{2}{|l|}{12 VS 16 months } \\
\hline Canonical Pathways & -log(p-value) & Canonical Pathways & $-\log (p$-value) \\
\hline Oxidative Phosphorylation & 17.3 & Oxidative Phosphorylation & 12.9 \\
\hline Mitochondrial Dysfunction & 16.6 & Mitochondrial Dysfunction & 11.1 \\
\hline EIF2 Signaling & 7.54 & Sirtuin Signaling Pathway & 6.32 \\
\hline Sirtuin Signaling Pathway & 5.58 & EIF2 Signaling & 4.39 \\
\hline TCA Cycle II (Eukaryotic) & 4.75 & Pyrimidine Deoxyribonucleotides De Novo Biosynthesis I & 3.17 \\
\hline
\end{tabular}

mice. The expression of Ctnnb1 was inhibited in F2 offspring [14]. This finding was further supported by various independent studies on the transgenerational inherited paternal acquired traits following mental stress [35], toxicant exposure [6], and diet changes [16]. Moreover, paternal age is another factor related to the health of offspring. Advanced paternal age in human increases the probability of genetic disorders such as schizophrenia, bipolar disorder, and autism, as reviewed by Sharma et al. [36]. Given that increased age generally also includes exposure to other complex environmental factors, such as physiological age, nutrition and lifestyle, genetics stability, hormones, senile diseases, oxidative stress, and telomere shortening [36], paternal effects of advanced age depend on both genetic and epigenetic changes. However, in our study of a relatively short age span in bulls (from 10 to 16 months old), epigenetic changes, especially sncRNAs, seemed to be affected by a young paternal age. In this study, fourteen miRNAs and one tsRNA were differentially expressed in sperm of younger bulls. Considering the vast amount of miRNAs stored in oocytes, sperm miRNAs that are also present in oocytes may have limited purposes, if any, in fertilization or early embryogenesis [37]. Thus, we finally selected ten miRNAs that were exclusively expressed in paternal gametes, based on published transcriptomic data of bovine oocytes $[20,21]$, to focus on specific sperm effects.

Several of the paternal differentially expressed sncRNAs in our study were associated with environmentally induced epigenetic inheritance of metabolic traits. A highfat and/or high-sugar diet (Western-like diet) induced obesity and type II diabetes in mice (F0) and these traits were inherited by the resulting progenies [38]. The expression of several miRNAs, including miR-19b was inhibited in sperm of F0 mice [38]. Intriguingly, when miR-19b was injected into wild type one-cell embryos, the offspring (here referred as R1) exhibited similar metabolic disorders as the F0 and F1 mice, and this pathological phenotype was further inherited after mating with healthy partners [38]. In another study focusing on mental stress, offspring of running males had suppressed reinstatement of juvenile fear memory; meanwhile, the expression of sperm-borne miR-19b and miR-133a was altered in these fathers, indicating the potential involvement of these miRNAs in the effects of paternal exercise on the anxiolytic behavioral phenotype of offspring [39].

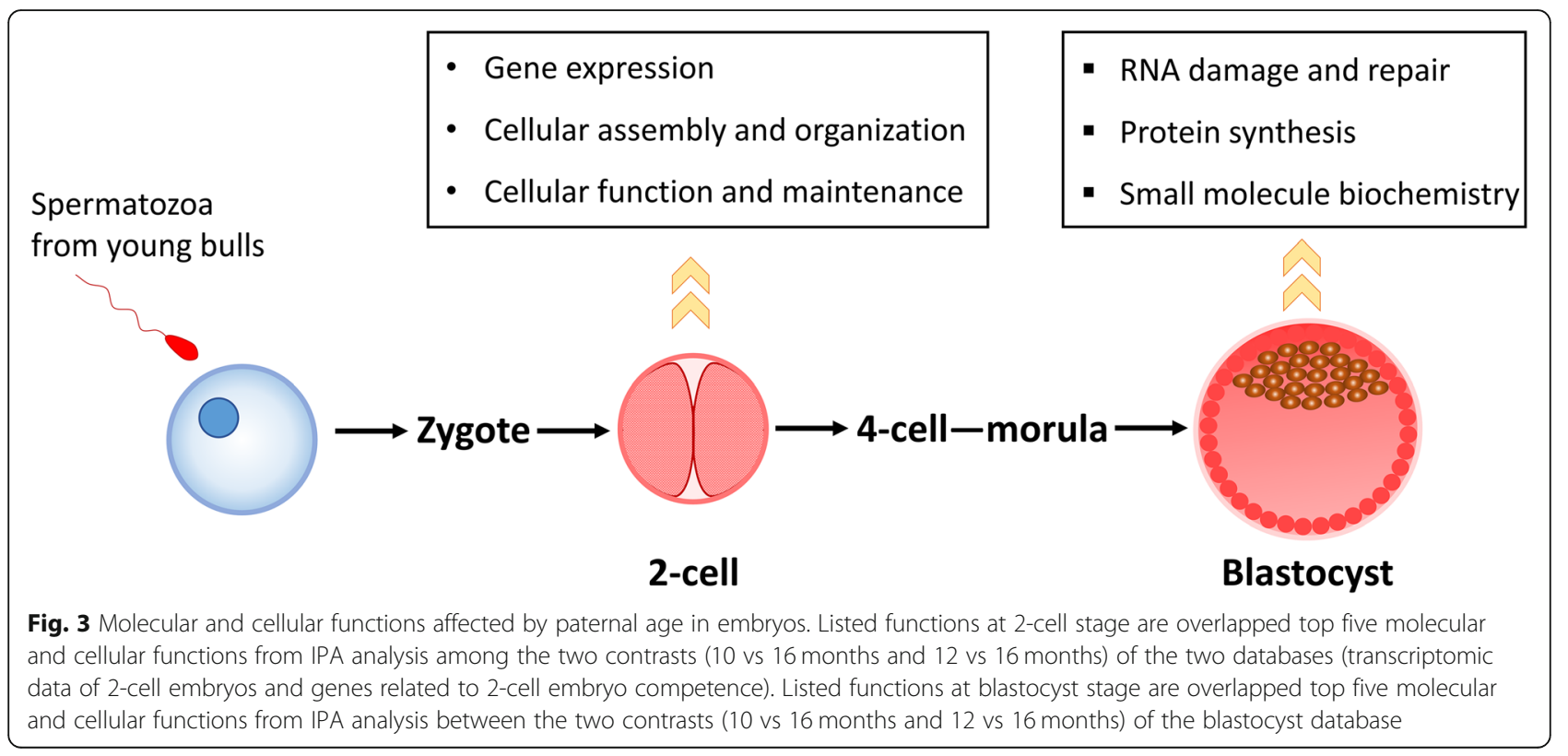




\section{Metabolism related pathways in early embryo development}

Normal development of the early embryo promotes further later normal development. For example, in bovine embryos, a faster cleavage of the zygote into a 2-cell embryo is positively correlated to a higher probability of the embryo becoming a viable blastocyst. In the present study, the transcriptome of bovine 2-cell embryos and genes related to developmental capacity were used to assess the value of target mRNA by sperm miRNA [23]. We then used a functional analysis (IPA) of the targets of paternal age-related sperm miRNAs to find pathways that might be affected. Although IPA were not specific to cattle, but the enrichment analysis results were similar to DAVID analysis (data not shown). Moreover, IPA provides an activation $\mathrm{z}$-score which infers to the activation status of biological function (activated or inhibited) [40]. Our results indicated that the pathways targeted were related to metabolism and totipotency which are immediately important for embryo quality and survival.

In the two contrasts, insulin-like growth factor 1 (IGF1) signaling was predicted to be activated by the interaction of sperm miRNA and maternal targets in 2-cell embryos generated from sperm from younger bulls (10 and 12 months), compared to 16-month-old bulls. Insulin-like growth factor 1 levels are higher in younger growing animals and possibly involved in metabolic programming [41-43]. The addition of physiological concentrations of IGF1 (10-100 ng/mL) improved the developmental competence of bovine early embryos in vitro [44-46]. In mice, a study using a high IGF1 concentration, i.e. 130 nM (201 $\mathrm{ng} / \mathrm{mL}$ ), which mimics the pathological condition of polycystic ovary syndrome, induced apoptosis in blastocysts via down regulation of the IGF1 receptor (IGF1R) [47] and a p53 dependant mechanism [48]. Similar results were also observed in bovine blastocysts supplemented with a supraphysiological concentration of IGF1 $(1000 \mathrm{ng} / \mathrm{mL})$, including induced apoptosis and decreased TP53 protein expression, but without inhibition of IGF1R [49]. Hence, the beneficial or adverse effects of IGF1 are concentration dependant and abnormally over activated IGF1 signaling might impair early embryogenesis.

Activation of TGF- $\beta$ signaling was predicted in sperm from 10 -month-old vs 16-month-old bulls. This pathway is involved in the regulation of several aspects of early embryonic development, such as embryonic patterning, cell fate determination, and dynamic movements [50]. The regulatory role of this pathway is exerted beyond the 2-cell stage in mouse embryos [51], indicating that it could possibly be programmed at the $2-8$ cell stage in bovine embryos and impact blastocyst differentiation. The Rho family GTPases, a family of small signaling G proteins, are involved in sperm induced egg activation [52]. In zygotes, inhibition of cell division cycle 42
(Cdc42), a member of the Rho family, significantly decreased in vitro development to the morula or blastocyst stage [53]. The involvement of Rho family GTPases in polarisation of early mouse blastomeres was also demonstrated [54].

The IPA predicted that several insulin-related pathways were impacted by paternal age. Insulin activates its receptor to phosphorylate and recruit many substrates, including IRS and Shc, to modulate various canonical pathways, such as the PI3K/Akt, AMPK, and Ras/MAPK pathways $[55,56]$. These pathways transduce the diverse effects of insulin on energy-associated cellular functions, such as glucose and lipid metabolism, protein synthesis, growth, and survival [57]. Although insulin transcripts are not detectable at the preimplantation stage in bovine embryos, the mRNA encoding insulin receptors, IGFI, and IGFII can be detected starting at the 1-cell stage [58], indicating that bovine embryos are sensitive to insulin. Similar to other mammals, cattle fed a restricted diet exhibited a reduced basal insulin concentration and improved pancreatic insulin sensitivity [59]. Hence, the insulin-related pathways predicted as activated in response to the different miRNAs in sperm from younger bulls might be associated with the prepubertal condition when energy is primarily devoted to growth, somewhat mimicking a feed restriction context. Transgenerational inheritance of paternal metabolic disorders was observed in various domestic farm animals [60]. As a consequence, embryos from younger bulls might have altered metabolism compared to embryos from adult bulls.

\section{Pre-implantation embryo developmental competence}

Transcriptome analysis of two-cell embryos with different developmental dynamics revealed that DEGs in the earlier or later cleaving embryos were related to the cell cycle, gene expression, RNA processing, and protein degradation functions [23]. Intriguingly, some of these DEGs were targets of the miRNAs related to bull age identified in the present study. The canonical pathway analysis of this dataset demonstrated that the IP3 pathway, PI3K pathway, and pluripotency related pathways could be impacted by paternal age and could therefore affect the developmental competence of two-cell embryos. The IP3 pathway has been identified as a common mechanism to activate the metabolism of unfertilized oocytes by inducing $\mathrm{Ca}^{2+}$ oscillations in various species, from marine invertebrates to mammals [61]. During bovine oocyte fertilization, the IP3 pathway is required for $\mathrm{Ca}^{2+}$ release, and its inhibition impairs the first embryonic interphase [62]. The PI3K pathway is widely studied as a mediator of cell growth, proliferation, differentiation, and survival signals. In mouse preimplantation embryos, the p85 and p110 subunits of PI3K were expressed from the one cell to the blastocyst stage [63]. 
Moreover, deletion of 3-phosphoinositide-dependent protein kinase 1 (PDK1) arrested mouse embryos at the two-cell stage as a result of the inhibition of embryonic genome activation (EGA) [64]. Pluripotency is the capacity of embryonic stem cells to differentiate into all cell types of a body and is controlled by several key regulators (OCT4, NANOG, SOX2, etc.). In bovine embryos, Oct4 and Sox 2 transcripts were present at the pre-EGA phase $[65,66]$. Meanwhile, during the maternal-toembryonic transition, transcripts of genes involved in the maintenance of pluripotency of embryos were also observed [67]. Hence, the paternal age-related miRNAs may potentially target pathways associated with two-cell competence to further impact early embryonic development.

In a previous study, we generated blastocysts in vitro with semen from different bulls of the same age as the ones used in the present study [29]. The transcriptome of these blastocysts was influenced by paternal age, and canonical pathways related to the DEGs resulted in altered energy production and protein synthesis. Due to their relatively short half life, miRNAs from sperm might not survive to the blastocyst stage. However, the predicted upstream regulators of DEGs identified in blastocysts could mediate their effects on the transcriptome of blastocysts. In this case, we found four and three upstream regulators that were the targets of differentially expressed miRNAs in the 10 vs 16 months and 12 vs 16 months contrasts. Intriguingly, when we identified the downstream targets of these regulators and redid the IPA analysis, the results of the small fraction were highly consistent with the overall DEGs of the blastocyst transcriptome associated with the age effect, indicating that sperm miRNAs could be directly involved in the intergenerational inheritance of factors associated with paternal age.

\section{Conclusion}

In summary, the analysis of sncRNA in sperm from bulls at different pubertal stages revealed that the abundance of specific miRNAs could be affected by paternal age and could potentially influence the metabolism and developmental capacity of pre-implantation embryos. This perspective calls for attention on the potential consequences of using gametes from younger animals; and the miRNAs identified in this study could potentially be used to assess if a bull is mature enough to be used commercially.

\section{Methods}

\section{Chemicals}

All reagents used in this study were of tissue culture grade and obtained from Sigma-Aldrich (Oakville, Ontario, Canada), unless otherwise specified.

\section{Semen collection}

All samples were obtained from The Semex Alliance (Guelph, ON, Canada), a commercial provider of bovine gametes. Semen was collected from each Holstein bull $(n=4$, bull \#03981, bull \#06622, and bull $\# 10051$, bull \#10085) at the ages of 10,12 , and 16 months using an artificial vagina, in compliance with the guidelines of the Canadian Council on Animal Care. Semen characteristics were examined and recorded and straws containing about $1.5 \times 10^{7}$ spermatozoa were frozen in liquid nitrogen. Each animal served as its own control and two contrasts (10 vs 16 months and 12 vs 16 months) were created for the RNA-seq analysis described below.

\section{Sperm small RNA extraction}

For each treatment, five straws of semen were thawed in $37^{\circ} \mathrm{C}$ water for $1 \mathrm{~min}$ and the content was combined. After washing twice with PBS, the pellets were resuspended $1 \mathrm{~mL}$ Somatic lysis buffer $(0.5 \%$ Triton X-100, PBS) and incubated on ice for 30 mins. After another wash with PBS, the pellets were resuspended in $60 \mu \mathrm{L}$ PBS and each sperm suspension was added to $33.3 \mu \mathrm{L}$ sperm lysis buffer (4M Guanidine Thiocyanate, 5\% Tween 20, 5\% Triton X-100, $120 \mathrm{mM}$ EDTA, and 120 $\mathrm{mM}$ Tris- $\mathrm{HCl} \mathrm{pH} 8.0), 3.3 \mu \mathrm{L}$ Proteinase $\mathrm{K}(20 \mathrm{mg} / \mathrm{mL})$, and $3.3 \mu \mathrm{L} 0.1 \mathrm{M}$ DTT, then incubated for $15 \mathrm{~min}$ at $60^{\circ} \mathrm{C}$ with gentle shaking $(20 \mathrm{rpm})$. Small RNAs were extracted with the miRNeasy Mini Kit (QIAGEN, Toronto, ON, Canada, Cat. \# 217004) following the manufacturer's instructions. Briefly, $700 \mu \mathrm{L}$ QIAzol lysis reagent were added to the previous mixture and then moved to a QIAshredder homogenizer column (QIAGEN, Toronto, ON, Canada, Cat. \# 79654). After centrifugation at 13,300 rpm for $2 \mathrm{~min}$ at RT, the flow through was transferred to a new tube and kept at RT for $5 \mathrm{~min}$ to further dissociate nucleoprotein complexes. Chloroform $(140 \mu \mathrm{L})$ was added and the samples were shaken vigorously for $15 \mathrm{~s}$. The samples were left at RT for $2-3 \mathrm{~min}$ and then centrifuged at $12,000 \mathrm{~g}$ for $15 \mathrm{~min}$ at $4{ }^{\circ} \mathrm{C}$. The upper aqueous phase of each sample was transferred to a new collection tube, 1.5 volume of absolute ethanol was added, and the solution was moved to an RNeasy Mini spin column to absorb the RNAs. The flow through was discarded after centrifugation and $40 \mu \mathrm{L}$ DNase buffer (27 Kunitz units) were added directly to the middle of the membrane in the column and then incubated at RT for $15 \mathrm{~min}$. The column was further washed with the provided buffer RWT once and buffer RPE twice. The column was placed into a new $2 \mathrm{~mL}$ collection tube and the RNAs were dissolved in $30 \mu \mathrm{L}$ elution buffer. The integrity and concentration of sperm RNA were evaluated with an Agilent 2100 Bioanalyzer (Agilent Technologies, Palo Alto, CA). 
Small RNA library construction and solexa sequencing The libraries of sperm small RNAs were constructed according to the manual of TruSeq Small RNA Library Prep Kit (Illumina, U.S.A., v02) with minor modifications. In brief, RNA $3^{\prime}$ and $5^{\prime}$ adapters were ligated to an average of $87 \mathrm{ng}$ of sperm RNA in $5 \mu \mathrm{l}$ nuclease-free water. The adapter-ligated RNA was then reverse transcribed in the presence of RNA RT Primer, first strand buffer, dNTP Mix, DTT, RNase inhibitor, and SupserScript II Reverse Transcriptase according to the protocol and incubated at $50{ }^{\circ} \mathrm{C}$ for $1 \mathrm{~h}$. For each sample, two primers: the same RNA PCR Primer 1 and a specific RNA PCR Primer index were used for 11 cycles of amplification. The amplified RNA libraries were resolved on a 6\% Novex TBE gel for $35 \mathrm{~min}$ at $145 \mathrm{~V}$, and DNA fragments ranging from 145 to $400 \mathrm{bp}$, which represented original RNA sizes of 20 to $275 \mathrm{bp}$, were cut and purified by ethanol precipitation. The pellets were dissolved in $10 \mu \mathrm{L}$ elution buffer $(10 \mathrm{mM}$ Tris- $\mathrm{HCl}, \mathrm{pH} 8.5)$ and the purity and concentration of the libraries were checked by NanoDrop. Sequencing was done on an Illumina Hiseq 2500 at McGill University (Montreal, QC, Canada) following the recommended protocol.

\section{Bioinformatics analysis of small RNAs}

Sequencing data were processed for differential expression and functional analysis. Reads were qualitytrimmed using Trimmomatic [68] before being aligned to a synthetic reference of all bovine miRNAs, tRNAs, and ncRNAs using bowtie. Differential expression was assessed using the DESeq [69] package. Differentially expressed miRNAs $(\geq 1.5$-fold change, $p$-value $\leq 0.05)$ that were reported to be expressed at high intensity in maternal gametes [20,21], which will dilute the original fold change of miRNAs in sperm under 1.2, were excluded. We therefore assumed that the remaining miRNAs were sperm-borne. Targets of these differentially expressed spermatic miRNAs were identified with TargetScan (release 7.2) [22]. Targets that are presented in both the of up- and down-regulated miRNAs were filtered out. The list of remaining targets was then overlapped with the transcriptomic databases from 2-cell embryos [23] and blastocysts [29]. For the functional prediction of sperm miRNAs in 2-cell embryos, databases of targets expressed at the 2-cell stage and related to 2-cell competence were created for IPA (Supplementary Materials, Tables S3, S4, S5, S6). For the functional analysis in blastocysts, upstream regulators that were predicted from the transcriptome data of blastocysts and were also targets of sperm miRNAs were selected. Downstream targets of these upstream regulators were extracted to create a new database for IPA analysis (Supplementary Materials, Tables S7 and S8).

\section{Statistical analyses}

One-way ANOVA with Turkey posttest analyses was performed on sperm count, concentration, total motility, and post-thaw progressive motility using GraphPad Prism 6 (GraphPad Software, San Diego, CA, USA). Differences were considered statistically significant with a $p$-value $<0.05$.

\section{Supplementary Information}

The online version contains supplementary material available at https://doi. org/10.1186/s12864-020-07206-5.

Additional file 1: Figure S1. Semen quality of bulls at age of 10, 12 and 16 months. A: Sperm count; B: Sperm concentration; C: Total motility; $D$ : Post-thaw progressive motility. Bars show the mean \pm standard error; different letters represent $p$-value $<0.05$.

Additional file 2: Figure S2. RNA patterns of sperm from four bulls at 10,12 , and 16-months old. A: Summary of bioanalyzer results of total RNA extracted from sperm. B: Summary of $6 \%$ Novex TBE gel results after libraries preparation. Adapters of $125 \mathrm{nt}$ were added, small noncoding RNAs as well as miRNAs were cut and purified for sequencing.

Additional file 3: Table S1. Total RNA extracted from bovine spermatozoa. Table S2. Differentially expressed miRNA in the two contrasts. Table S3. Dataset of targets expressed in 2-cell embryo of 10vs16 months contrast for IPA. Table S4. Dataset of targets expressed in 2-cell embryo of 12vs16 months contrast for IPA. Table S5. Dataset of targets related to 2-cell embryo competence of 10vs16 months contrast for IPA. Table S6. Dataset of targets related to 2-cell embryo competence of 12 vs 16 months contrast for IPA. Table S7. Dataset of targets expressed in blastocyst of 10vs16 months contrast for IPA. Table S8. Dataset of targets expressed in blastocyst of 12vs16 months contrast for IPA.

\section{Abbreviations}

DEGs: Differentially expressed genes; DMRs: Differentially methylated regions; IGF1: Insulin like growth factor 1; IGF1R: Insulin like growth factor 1 receptor; IPA: Ingenuity pathway analysis; miRNA: microRNA; sncRNAs: Small noncoding RNAs; tsRNA: Transfer RNA-derived small RNAs

\section{Acknowledgements}

The authors would like to thank Isabelle Dufort for technical support, Eric Fournier for assistance of data processing, and Sylvie Bilodeau-Goeseels for proofreading this article.

Authors' contributions

MAS, PB and CW conceived and designed the experiments. Christian Vigneault and Rémi Labrecque prepared the semen samples. CW extracted performed the library and data analyses. CW wrote the manuscript, reviewed by MAS and PB. All authors read and approved the final manuscript.

\section{Funding}

This work was funded by the Natural Sciences and Engineering Research Council (NSERC grant no 445230-12), Semex and DairyGen of Canada. Chongyang Wu received studentship support from China Scholarship Council-Université Laval (CSC-UL) joint scholarship.

\section{Availability of data and materials}

RNA-seq data were deposited in NCBI's Gene Expression Omnibus (GEO) and are accessible through GEO series accession number: GSE147742 (https:// www.ncbi.nlm.nih.gov/geo/query/acc.cgi?acc=GSE147742).

\section{Ethics approval and consent to participate}

All samples were collected in compliance with the guidelines of the Canadian Council on Animal Care. These guidelines are strictly followed by the local abattoir and L'Alliance Boviteq who provided all the tissues and samples. The study did not require handling animals on university premises. 


\section{Consent for publication}

Not applicable.

\section{Competing interests}

The authors declare no conflict of interests.

\section{Author details}

${ }^{1}$ Centre de Recherche en Reproduction, Développement et Santé Intergénérationnelle (CRDSI), Département des Sciences Animales, Faculté des Sciences de I'Agriculture et de l'Alimentation, Université Laval, Québec, Québec, Canada. 'L'Alliance Boviteq, Saint-Hyacinthe, Québec, Canada.

Received: 14 April 2020 Accepted: 29 October 2020

Published online: 16 November 2020

\section{References}

1. Hayes BJ, Bowman PJ, Chamberlain AJ, Goddard ME. Invited review: genomic selection in dairy cattle: Progress and challenges. J Dairy Sci. 2009; 92(2):433-43.

2. Wolf F, Almquist J, Hale E. Prepuberal behavior and puberal characteristics of beef bulls on high nutrient allowance. J Anim Sci. 1965;24(3):761-5.

3. Takeda K, Kobayashi E, Akagi S, Nishino K, Kaneda M, Watanabe S. Differentially methylated $\mathrm{CPG}$ sites in bull spermatozoa revealed by human DNA methylation arrays and bisulfite analysis. J Reprod Dev. 2017;63(3):279-87.

4. Okada Y, Yamaguchi K. Epigenetic modifications and reprogramming in paternal pronucleus: sperm, preimplantation embryo, and beyond. Cell Mol Life Sci. 2017;74(11):1957-67.

5. Siklenka K, Erkek S, Godmann M, Lambrot R, McGraw S, Lafleur C, et al. Disruption of histone methylation in developing sperm impairs offspring health transgenerationally. Science. 2015;350(6261):aab2006.

6. Skinner MK, Ben Maamar M, Sadler-Riggleman I, Beck D, Nilsson E, McBirney $M$, et al. Alterations in sperm DNA methylation, non-coding RNA and histone retention associate with DDT-induced epigenetic transgenerational inheritance of disease. Epigenetics Chromatin. 2018;11(1):8.

7. Govindaraju A, Uzun A, Robertson L, Atli MO, Kaya A, Topper E, et al. Dynamics of microRNAs in bull spermatozoa. Reprod Biol Endocrinol. 2012; 10(1):82.

8. Fagerlind M, Stålhammar H, Olsson B, Klinga-Levan K. Expression of miRNAs in bull spermatozoa correlates with fertility rates. Reprod Domest Anim. 2015:50(4):587-94.

9. Kutchy NA, Menezes ESB, Chiappetta A, Tan W, Wills RW, Kaya A, et al. Acetylation and methylation of sperm histone 3 lysine 27 (H3K27ac and H3K27me3) are associated with bull fertility. Andrologia. 2018;50(3):e12915.

10. Ugur MR, Kutchy NA, de Menezes EB, Ul-Husna A, Haynes BP, Uzun A, et al. Retained acetylated histone four in bull sperm associated with fertility. Front Vet Sci. 2019;6:223.

11. Lambert S, Blondin P, Vigneault C, Labrecque R, Dufort I, Sirard M-A. Spermatozoa DNA methylation patterns differ due to peripubertal age in bulls. Theriogenology. 2018;106(Supplement C):21-9.

12. Takeda K, Kobayashi E, Nishino K, Imai A, Adachi H, Hoshino Y, et al. Agerelated changes in DNA methylation levels at $\mathrm{CpG}$ sites in bull spermatozoa and in vitro fertilization-derived blastocyst-stage embryos revealed by combined bisulfite restriction analysis. J Reprod Dev. 2019;65(4):305-12.

13. Fullston T, Ohlsson Teague EMC, Palmer NO, DeBlasio MJ, Mitchell M, Corbett $\mathrm{M}$, et al. Paternal obesity initiates metabolic disturbances in two generations of mice with incomplete penetrance to the F2 generation and alters the transcriptional profile of testis and sperm microRNA content. FASEB J. 2013;27(10):4226-43.

14. Gapp K, Jawaid A, Sarkies P, Bohacek J, Pelczar P, Prados J, et al. Implication of sperm RNAs in transgenerational inheritance of the effects of early trauma in mice. Nat Neurosci. 2014;17:667.

15. Sharma U, Conine CC, Shea JM, Boskovic A, Derr AG, Bing XY, et al. Biogenesis and function of tRNA fragments during sperm maturation and fertilization in mammals. Science. 2016;351(6271):391-6.

16. Chen $Q$, Yan M, Cao Z, Li X, Zhang Y, Shi J, et al. Sperm tsRNAs contribute to intergenerational inheritance of an acquired metabolic disorder. Science. 2016;351(6271):397-400.

17. Yuan S, Schuster A, Tang C, Yu T, Ortogero N, Bao J, et al. Sperm-borne miRNAs and endo-siRNAs are important for fertilization and preimplantation embryonic development. Development. 2016;143(4):635-47.
18. Liu WM, Pang RTK, Chiu PCN, Wong BPC, Lao KQ, Lee KF, et al. Sperm-borne microRNA-34c is required for the first cleavage division in mouse. Proc Natl Acad Sci U S A. 2012;109(2):490-4.

19. Du Y, Wang $X$, Wang B, Chen W, He R, Zhang L, et al. Deep sequencing analysis of microRNAs in bovine sperm. Mol Reprod Dev. 2014;81(11):1042-52.

20. Gilchrist GC, Tscherner A, Nalpathamkalam T, Merico D, LaMarre J. MicroRNA expression during bovine oocyte maturation and fertilization. Int J Mol Sci. 2016;17(3):396.

21. Andrade G, Meirelles F, Perecin F, da Silveira J. Cellular and extracellular vesicular origins of miRNAs within the bovine ovarian follicle. Reprod Domest Anim. 2017;52(6):1036-45.

22. Agarwal V, Bell GW, Nam J-W, Bartel DP. Predicting effective microRNA target sites in mammalian mRNAs. Elife. 2015;4:e05005.

23. Orozco-Lucero E, Dufort I, Robert C, Sirard MA. Rapidly cleaving bovine twocell embryos have better developmental potential and a distinctive mRNA pattern. Mol Reprod Dev. 2014;81(1):31-41.

24. Dode MAN, Dufort I, Massicotte L, Sirard MA. Quantitative expression of candidate genes for developmental competence in bovine two-cell embryos. Mol Reprod Dev. 2006;73(3):288-97.

25. Lonergan $\mathrm{P}$, Khatir H, Piumi F, Rieger D, Humblot P, Boland MP. Effect of time interval from insemination to first cleavage on the developmental characteristics, sex ratio and pregnancy rate after transfer of bovine embryos. Reproduction. 1999;117(1):159-67.

26. Memili E, First NL. Zygotic and embryonic gene expression in cow: a review of timing and mechanisms of early gene expression as compared with other species. Zygote. 2000;8(1):87-96.

27. Kues WA, Sudheer S, Herrmann D, Carnwath JW, Havlicek V, Besenfelder U, et al. Genome-wide expression profiling reveals distinct clusters of transcriptional regulation during bovine preimplantation development in vivo. Proc Natl Acad Sci U S A. 2008;105(50):19768-73.

28. Tscherner A, Gilchrist G, Smith N, Blondin P, Gillis D, LaMarre J. MicroRNA-34 family expression in bovine gametes and preimplantation embryos. Reprod Biol Endocrinol. 2014;12(1):85.

29. Wu C, Blondin P, Vigneault C, Labrecque R, Sirard M-A. The age of the bul influences the transcriptome and epigenome of blastocysts produced by IVF. Theriogenology. 2020;144:122-31.

30. Yuan S, Tang C, Zhang Y, Wu J, Bao J, Zheng H, et al. Mir-34b/C and mir$449 \mathrm{a} / \mathrm{b} / \mathrm{c}$ are required for spermatogenesis, but not for the first cleavage division in mice. Biol Open. 2015;4(2):212-23.

31. Rando OJ. Daddy issues: paternal effects on phenotype. Cell. 2012;151(4): 702-8.

32. Wang L, Zhang J, Duan J, Gao X, Zhu W, Lu X, et al. Programming and inheritance of parental DNA methylomes in mammals. Cell. 2014;157(4): 979-91.

33. Radford EJ, Ito M, Shi H, Corish JA, Yamazawa K, Isganaitis E, et al. In utero undernourishment perturbs the adult sperm methylome and intergenerational metabolism. Science. 2014;345(6198):1255903.

34. Shea Jeremy M, Serra Ryan W, Carone Benjamin R, Shulha Hennady P, Kucukural A, Ziller Michael J, et al. Genetic and epigenetic variation, but not diet. Shape Sperm Methylome Dev Cell. 2015;35(6):750-8.

35. Rodgers AB, Morgan CP, Leu NA, Bale TL. Transgenerational epigenetic programming via sperm microRNA recapitulates effects of paternal stress. Proc Natl Acad Sci U S A. 2015;112(44):13699-704.

36. Sharma R, Agarwal A, Rohra VK, Assidi M, Abu-Elmagd M, Turki RF. Effects of increased paternal age on sperm quality, reproductive outcome and associated epigenetic risks to offspring. Reprod Biol Endocrinol. 2015;13(1):35.

37. Amanai M, Brahmajosyula M, Perry ACF. A restricted role for sperm-borne MicroRNAs in mammalian Fertilization1. Biol Reprod. 2006;75(6):877-84.

38. Grandjean V, Fourré S, De Abreu DAF, Derieppe M-A, Remy J-J, Rassoulzadegan M. RNA-mediated paternal heredity of diet-induced obesity and metabolic disorders. Sci Rep. 2015;5(1):18193.

39. Short A, Yeshurun S, Powell R, Perreau V, Fox A, Kim J, et al. Exercise alters mouse sperm small noncoding RNAs and induces a transgenerational modification of male offspring conditioned fear and anxiety. Transl Psychiatry. 2017;7(5):e1114.

40. Krämer A, Green J, Pollard J Jr, Tugendreich S. Causal analysis approaches in ingenuity pathway analysis. Bioinformatics. 2013;30(4):523-30.

41. Breese CR, Ingram RL, Sonntag WE. Influence of age and long-term dietary restriction on plasma insulin-like growth factor-1 (IGF-1), IGF-1 gene expression, and IGF-1 binding proteins. J Gerontol. 1991;46(5):B180-7. 
42. Greer KA, Hughes LM, Masternak MM. Connecting serum IGF-1, body size, and age in the domestic dog. AGE. 2011;33(3):475-83.

43. Bourgon SL, Diel de Amorim M, Miller SP, Montanholi YR. Associations of blood parameters with age, feed efficiency and sampling routine in young beef bulls. Livest Sci. 2017;195:27-37.

44. Matsui M, Takahashi $Y$, Hishinuma M, Kanagawa H. Insulin and insulin-like growth factor-I (IGF-I) stimulate the development of bovine embryos fertilized in vitro. J Vet Med Sci. 1995;57(6):1109-11.

45. Sirisathien S, Hernandez-Fonseca HJ, Brackett BG. Influences of epidermal growth factor and insulin-like growth factor-I on bovine blastocyst development in vitro. Anim Reprod Sci. 2003;77(1-2):21-32.

46. Tríbulo P, Jumatayeva G, Lehloenya K, Moss Il, Negrón-Pérez VM, Hansen PJ. Effects of sex on response of the bovine preimplantation embryo to insulinlike growth factor 1, activin A, and WNT7A. BMC Dev Biol. 2018;18(1):16.

47. Chi MM, Schlein AL, Moley KH. High insulin-like growth factor 1 (IGF-1) and insulin concentrations trigger apoptosis in the mouse blastocyst via downregulation of the IGF-1 receptor. Endocrinology. 2000;141(12):4784-92.

48. Moley KH, Bibee K, Wyman A, Eng GS. IGF-1 induced blastocyst apoptosis is p53 dependent. Fertil Steril. 2005;84:S388.

49. Velazquez MA, Hermann D, Kues WA, Niemann H. Increased apoptosis in bovine blastocysts exposed to high levels of IGF1 is not associated with downregulation of the IGF1 receptor. Reproduction. 2011;141(1):91-103.

50. Liu $C$, Peng G, Jing N. TGF- $\beta$ signaling pathway in early mouse development and embryonic stem cells. Acta Biochim Biophys Sin. 2017; 50(1):68-73.

51. Roelen BAJ, Goumans M-J, Zwijsen A, Mummery CL. Identification of two distinct functions for TGF- $\beta$ in early mouse development. Differentiation. 1998:64(1):19-31.

52. Moore GD, Ayabe T, Visconti PE, Schultz RM, Kopf GS. Roles of heterotrimeric and monomeric $\mathrm{G}$ proteins in sperm-induced activation of mouse eggs. Development. 1994;120(11):3313-23.

53. Cui X-S, Li X-Y, Kim N-H. Cdc42 is implicated in polarity during meiotic resumption and blastocyst formation in the mouse. Mol Reprod Dev. 2007; 74(6):785-94.

54. Clayton L, Hall A, Johnson MH. A role for rho-like GTPases in the polarisation of mouse eight-cell Blastomeres. Dev Biol. 1999:205(2):322-31.

55. Mayer JP, Zhang F, DiMarchi RD. Insulin structure and function. Biopolymers. 2007:88(5):687-713.

56. Siddle K. Signalling by insulin and IGF receptors: supporting acts and new players. J Mol Endocrinol. 2011:47(1):R1-10.

57. Guo S. Insulin signaling, resistance, and the metabolic syndrome: insights from mouse models into disease mechanisms. J Endocrinol. 2014;220(2):T1-t23.

58. Schultz GA, Hogan A, Watson AJ, Smith RM, Heyner S. Insulin, insulin-like growth factors and glucose transporters: temporal patterns of gene expression in early murine and bovine embryos. Reprod Fertil Dev. 1992;4(4):361-71.

59. Keogh K, Kenny DA, Kelly AK, Waters SM. Insulin secretion and signaling in response to dietary restriction and subsequent re-alimentation in cattle. Physiol Genomics. 2015;47(8):344-54.

60. Thompson RP, Nilsson E, Skinner MK. Environmental epigenetics and epigenetic inheritance in domestic farm animals. Anim Reprod Sci. 2020;106316.

61. Ciapa B, Chiri S. Egg activation: upstream of the fertilization calcium signal. Biol Cell. 2000;92(3-4):215-33.

62. Malcuit C, Knott JG, He C, Wainwright T, Parys JB, Robl JM, et al. Fertilization and inositol 1,4,5-Trisphosphate (IP3)-induced calcium release in Type-1 inositol 1,4,5-Trisphosphate receptor Down-regulated bovine Eggs1. Biol Reprod. 2005;73(1):2-13.

63. Riley JK, Carayannopoulos MO, Wyman AH, Chi M, Ratajczak CK, Moley KH. The PI3K/Akt pathway is present and functional in the preimplantation mouse embryo. Dev Biol. 2005;284(2):377-86.

64. Zheng W, Gorre N, Shen Y, Noda T, Ogawa W, Lundin E, et al. Maternal phosphatidylinositol 3-kinase signalling is crucial for embryonic genome activation and preimplantation embryogenesis. EMBO Rep. 2010;11(11):890-5.

65. Kurosaka S, Eckardt S, McLaughlin KJ. Pluripotent lineage definition in bovine embryos by Oct4 transcript Localization1. Biol Reprod. 2004;71(5): 1578-82.

66. Khan DR, Dubé D, Gall L, Peynot N, Ruffini S, Laffont L, et al. Expression of Pluripotency master regulators during two key developmental transitions: EGA and early lineage specification in the bovine embryo. PLoS One. 2012;7(3):1-12.

67. Vigneault C, Gravel C, Vallée M, McGraw S, Sirard M-A. Unveiling the bovine embryo transcriptome during the maternal-to-embryonic transition. Reproduction. 2009;137(2):245.
68. Bolger AM, Lohse M, Usadel B. Trimmomatic: a flexible trimmer for Illumina sequence data. Bioinformatics. 2014;30(15):2114-20.

69. Anders S, Huber W. Differential expression analysis for sequence count data. Genome Biol. 2010;11(10):R106

\section{Publisher's Note}

Springer Nature remains neutral with regard to jurisdictional claims in published maps and institutional affiliations.
Ready to submit your research? Choose BMC and benefit from:

- fast, convenient online submission

- thorough peer review by experienced researchers in your field

- rapid publication on acceptance

- support for research data, including large and complex data types

- gold Open Access which fosters wider collaboration and increased citations

- maximum visibility for your research: over $100 \mathrm{M}$ website views per year

At BMC, research is always in progress.

Learn more biomedcentral.com/submissions 